\title{
Choose Best Criteria for Decision Making Via Fuzzy Topsis Method
}

\author{
Muhammad Zulqarnain, Fazal Dayan* \\ Department of Mathematics, University of Management and Technology, Lahore, Pakistan \\ Email address: \\ fazaldayan1@gmail.com (F. Dayan) \\ ${ }^{*}$ Corresponding author \\ To cite this article: \\ Muhammad Zulqarnain, Fazal Dayan. Choose Best Criteria for Decision Making Via Fuzzy Topsis Method. Mathematics and Computer \\ Science. Vol. 2, No. 6, 2017, pp. 113-119. doi: 10.11648/j.mcs.20170206.14
}

Received: October 10, 2017; Accepted: October 27, 2017; Published: November 24, 2017

\begin{abstract}
Multi Criteria Decision Making (MCDM) uses different techniques to find a best alternative from multialternative and multi-criteria conditions. TOPSIS is an important practical technique for ranking and selection of different alternatives by using distance measures. Classical TOPSIS uses crisp techniques for the linguistic assessments, but due to imprecise and fuzziness nature of the linguistic assessments, we faced some problems to find out the solution of these problems. We proposed a Fuzzy TOPSIS with its example in this work and use this method for decision making.
\end{abstract}

Keywords: Fuzzy TOPSIS, Fuzzy Set, Aggregated Fuzzy Decision Matrix, Normalized Fuzzy Decision Matrix, Weighted Normalized Fuzzy Decision Matrix

\section{Introduction}

Hwang and Yoon invented the Technique for Order Preference by Similarity to Ideal Solution (TOPSIS) in order to solve MCDM problem with many alternatives [1]. From crisp to fuzzy data, Chen \& Hwang remodelled TOPSIS [2]. Furthermore, Chen widened the TOPSIS for Group Decision Making in fuzzy atmosphere [3]. Awasthi et al. used fuzzy TOPSIS for the evaluation and selection of the best location planning for urban distribution centres [4]. Chu [5] and Yong [6] applied fuzzy TOPSIS for choosing plant location with minimum costs and maximum use of resources. The relative closeness coefficients were obtained as fuzzy numbers and after defuzzification, alternatives were ranked [7]. Wang and Elhang found that their method is much closer to the fuzzy weighted approach presented by Dong and Wong [8]. Mahmoodzadeh et al. incorporating fuzzy AHP and TOPSIS method gave a new procedure for the project selection problem. Improved fuzzy AHP was used to compute the weights of each criterion at first and then TOPSIS algorithm was engaged for ranking the projects to be selected [9].

Yong Tsao et al. utilized fuzzy TOPSIS for supporting contractors to choose suitable project for bidding with the help of MAGDM. Triangular fuzzy numbers (TFNs) were assigned to each linguistic variable for alternative ratings and criteria weights [10]. Zadeh proposed Type-2 FS to encompass uncertainty about the membership function in fuzzy set theory (FST) [11]. Saremi and Montazer, to cope with the decision-making problems having data with large uncertainty, used a Type-2 fuzzy TOPSIS based method. A Study of TOPSIS in Classical, Fuzzy, Intuitionistic Fuzzy and Neutrosophic Environments Interval-valued fuzzy (IVF) set is a special type of Type-2 FS. Saremi and Montazer applied IVF sets having lower and upper triangular membership functions; they ranked the alternatives [12]. Chen and Tsao broadened IVF-TOPSIS to solve MADM problem [13].

Zulqarnain. M. and Saeed. M. proposed and proved the credibility of interval valued fuzzy soft matrix (IVFSM) in decision making. They discussed its different properties [14]. They also studied fuzzy soft matrix (FSM) and IVFSM and redefined the product of IVFSM. Later they used IVFSM and FSM in decision making problem with examples and compare the results. They observed that FSM method is more appropriate for decision making [15]. They proposed a new decision making method on IVFSM named as "interval valued fuzzy soft max-min decision making method" with the help of interval valued fuzzy soft max-min decision making function and used this method for decision making [16]. 


\section{Prelimnires}

In this section, we discussed about FS and fuzzy TOPSIS which is proposed by (Zadeh 1965) with examples and some important propositions.

\subsection{Definition 1 [22]}

If $A$ be a set and $U$ be a universal set. Set $A$ contains different elements of set $U$ then we assign all elements of $A$ any membership value between the interval $[0,1]$ is called FS.

\subsection{Definition 2 [18]}

A fuzzy number is a convex and normal fuzzy subset of the universe of discourse $\mathrm{X}$.

\subsection{Definition 3 [22]}

If $A$ and $B$ are two FS than $A$ is called fuzzy subset of $B$ if $\mu_{\mathrm{A}}(\mathrm{t}) \leq \mu_{\mathrm{B}}(\mathrm{t})$ for all $\mathrm{t} \in \mathrm{U}$. It can be represented as $\mathrm{A} \leq \mathrm{B}$.

\subsection{Example 2}

Let $\mathrm{U}=\left\{\mathrm{P}_{1}, \mathrm{P}_{2}, \mathrm{P}_{3}, \mathrm{P}_{4}, \mathrm{P}_{5}, \mathrm{P}_{6}, \mathrm{P}_{7}, \mathrm{P}_{8}\right\}$ be a universal set, $\mathrm{A}$ and $\mathrm{B}$ are any two FS such that $\mathrm{A}$ is subset of $\mathrm{B}$. Let

$$
\begin{aligned}
\mathrm{A} & =\left\{\mathrm{P}_{1}, \mathrm{P}_{3}, \mathrm{P}_{4}, \mathrm{P}_{5}, \mathrm{P}_{6}, \mathrm{P}_{7}\right\}, \mathrm{B}=\left\{\mathrm{P}_{1}, \mathrm{P}_{2}, \mathrm{P}_{3}, \mathrm{P}_{4}, \mathrm{P}_{5}, \mathrm{P}_{6}, \mathrm{P}_{7}\right\}, \mathrm{f}_{\mathrm{A}} \\
& =\mu_{\mathrm{A}}(\mathrm{t})=\left\{\mathrm{P}_{1} / 0.3, \mathrm{P}_{3} / 0.1 . \mathrm{P}_{4} / 0.7, \mathrm{P}_{5} / 0.4, \mathrm{P}_{6} / 0.9, \mathrm{P}_{7} / 0.2\right\}
\end{aligned}
$$

and $\mathrm{f}_{\mathrm{B}}=\mu_{\mathrm{B}}(\mathrm{t})=\left\{\mathrm{P}_{1} / 0.3, \mathrm{P}_{2} / 0.2, \mathrm{P}_{3} / 0.2, \mathrm{P}_{4} / 0.9, \mathrm{P}_{5} / 0.6\right.$, $\left.\mathrm{P}_{6} / 0.9, \mathrm{P}_{7} / 0.3\right\}$.

Clearly $\mu_{\mathrm{A}}(\mathrm{t})$ is subset of $\mu_{\mathrm{B}}(\mathrm{t})$ for all $\mathrm{t} \in \mathrm{U}$, therefore $A \subseteq B$.

\subsection{Definition 3 [22]}

A FS in which $\mu_{\mathrm{A}}(\mathrm{t})=0$ for all $\mathrm{t} \in \mathrm{U}$ is called empty FS.

\subsection{Definition 4 [22]} FS.

A FS in which $\mu_{\mathrm{A}}(\mathrm{t})=1$ for all $\mathrm{t} \in \mathrm{U}$ is called universal

\subsection{Definition 5 [22]}

If $\mathrm{A}$ and $\mathrm{B}$ are two FS than they are called equal FS if $\mu_{\mathrm{A}}$ $(t)=\mu_{B}(t)$ for all $t \in U$. It can be represented as $A=B$.

\subsection{Definition 6 [22]}

Let $\mathrm{A}$ be a FS than its complement is defined as

$$
\mathrm{f}_{A}{ }^{\mathrm{c}}(\mathrm{t})=1-\mathrm{f}_{\mathrm{A}}(\mathrm{t}) \text { for all } \mathrm{t} \in \mathrm{U} \text {. }
$$

\subsection{Definition 7 [22]}

If $\mathrm{A}$ and $\mathrm{B}$ are two FS whose membership functions are $\mu_{\mathrm{A}}$ (t) and $\mu_{B}(t)$ respectively, than their union is also a FS, i.e. $\mathrm{C}=\mathrm{A} \mathrm{U} \mathrm{B}$ which is defined as

$$
\mathrm{f}_{\mathrm{C}}(\mathrm{t})=\max \left(\mu_{\mathrm{A}}(\mathrm{t}), \mu_{\mathrm{B}}(\mathrm{t})\right) \text { for all } \mathrm{t} \in \mathrm{U} \text {. }
$$

\subsection{Definition 8 [22]}

If $\mathrm{A}$ and $\mathrm{B}$ are two FS whose membership functions are $\mu_{\mathrm{A}}$ (t) and $\mu_{B}(t)$ respectively, than their intersection is also a FS, i.e.

$\mathrm{C}=\mathrm{A} \cap \mathrm{B}$ which is defined as

$$
\mathrm{f}_{\mathrm{C}}(\mathrm{t})=\min \left(\mu_{\mathrm{A}}(\mathrm{t}), \mu_{\mathrm{B}}(\mathrm{t})\right) \text { for all } \mathrm{t} \in \mathrm{U} \text {. }
$$

\section{Fuzzy Topsis Algorithm}

In order to deal with data and information containing nonstatistical uncertainties, the best tool is the theory of FS which was proposed by Zadeh. It is a matter of fact that we are not always in a position to express our viewpoint exactly. Many opinions are only unclear and uncertain. To model such scenarios more accurately, in 1965 Zadeh proposed a new theory, named as FST [19]. As far as fuzzy TOPSIS is concerned, Chen extended TOPSIS to Fuzzy-TOPSIS using TFNs to replace the numeric linguistic scales for rating the alternatives and to assign the weights to the criteria [20].

One of the major differences between Fuzzy-TOPSIS and Classical-TOPSIS is that the later utilizes precisely known ratings and weights of the criteria [21]. Fuzzy-TOPSIS is an application of Fuzzy logic and FS. The Algorithm of Fuzzy TOPSIS is given below.

Consider a set of "m" Alternatives $\mathrm{A}=$ $\left\{A_{1}, A_{2}, A_{3}, . ., A_{m}\right\}$, a set of "n" Evaluation Criteria, $\mathrm{C}=$ $\left\{C_{1}, C_{2}, C_{3}, . ., C_{m}\right\}$ and a set of "i" Decision Makers DMs = $\left\{\mathrm{DM}_{1}, \mathrm{DM}_{2}, \ldots, \mathrm{DM}_{\mathrm{i}}\right\}$.

Step 1:

Selection of a fuzzy rating scale for linguistic variables, since alternatives as well as criteria both are in the form of linguistic variables.

Step 2:

Criteria weightage and fuzzy linguistic ratings for the alternatives given by DM's Taking $\check{x}_{i j}^{k}$ as fuzzy ratings by $k^{\text {th }}$ decision maker for the $i^{\text {th }}$ alternative w. r. t the $j^{\text {th }}$ criterion, represented by the following equation.

$$
\check{x}_{i j}^{k}=\left(a_{i j}^{k}, b_{i j}^{k}, c_{i j}^{k}\right)
$$

And $w_{j}^{k}$ is the weight allocated by the $k^{\text {th }}$ decision maker for the $j^{\text {th }}$ criterion, represented by the equation given below.

$$
w_{j}^{k}=\left\{w_{j 1}^{k}, w_{j 2}^{k}, w_{j 3}^{k}\right\}
$$

Step 3:

Compute the aggregated fuzzy ratings for the alternatives, taking $\breve{x}_{i j}$ as aggregated fuzzy ratings for the $i^{\text {th }}$ alternative $\mathrm{w}$. r. t the $j^{\text {th }}$ criterion,

$$
\check{x}_{i j}=\left(a_{i j}, b_{i j}, c_{i j}\right), a_{i j}=\min _{k}\left\{a_{i j}^{k}\right\}, b_{i j}=\frac{1}{l} \sum_{k=1}^{l} b_{i j}^{k}, c_{i j}=
$$

and $w_{j}$ is the aggregated fuzzy weights for the $j^{\text {th }}$ criterion.

$$
w_{i j}=\left(a_{j 1}, b_{j 2}, c_{j 3}\right), w_{i j}=\min _{k}\left\{w_{j 1}^{k}\right\}, w_{i j}=\frac{1}{l} \sum_{k=1}^{l} w_{j 2}^{k}, w_{i j}=
$$




$$
\underset{k}{\max }\left\{w_{j 3}^{k}\right\}
$$

Step 4: Construction of AFDM and Aggregated Fuzzy Weight Matrix (AFWM).

Now the Fuzzy MCDM problem will be converted to an AFDM as given below

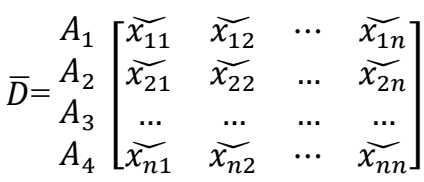

where $x_{i j}$ is aggregated fuzzy rating for the $i^{\text {th }}$ alternative w. r. $\mathrm{t}$ the $j^{\text {th }}$ criterion. Moreover, AFWM is defined as

$$
\grave{W}=\left[\dot{w}_{1}, \dot{w}_{2}, \dot{w}_{3}, \ldots \ldots \ldots . . . \dot{w}_{n}\right]^{\text {transpose }}
$$

where $\dot{w}_{j}$, is the aggregated fuzzy weight of $j^{\text {th }}$ criterion.

Step 5: Normalization of the FDM:

The NFDM is given below

$$
\bar{R}=\left[\dot{r}_{i j}\right]_{\mathrm{m} \times \mathrm{n}}=\left[\begin{array}{cccc}
\widetilde{r_{11}} & \widetilde{r_{12}} & \cdots & \widetilde{r_{1 n}} \\
\widetilde{r_{21}} & \overrightarrow{r_{22}} & \cdots & \overline{r_{2 n}} \\
\cdots & \cdots & \cdots & \cdots \\
\widetilde{r_{m 1}} & \widetilde{r_{m 2}} & \cdots & \widetilde{r_{m n}}
\end{array}\right]
$$

where $\dot{r}_{i j}=\left(\frac{a_{i j}}{c_{j}^{*}}, \frac{b_{i j}}{c_{j}^{*}}, \frac{c_{i j}}{c_{j}^{*}}\right), c_{j}^{*}=\max _{i} c_{i j}$ (benefit criteria), $\dot{r}_{i j}=\left(\frac{a_{j}^{-}}{c_{i j}}, \frac{a_{j}^{-}}{b_{i j}}, \frac{a_{j}^{-}}{a_{i j}}\right)$ and $a_{j}^{-}=\min _{i} a_{i j}$ (cost criteria)

The Linear Scale Transformation (LST) is used to normalize the Decision Matrix. The important point of this normalization method is that the normalized TFN are within the interval $[0,1]$.

Step 6: WNFDM

The WNFDM $\bar{V}=$ is given below

$$
\begin{gathered}
\bar{V}=\left[\hat{v}_{i j}\right]_{\mathrm{m} \times \mathrm{n}}=\left[\dot{w}_{j}(.) \dot{r}_{i j}\right]= \\
{\left[\begin{array}{cccc}
w_{1}(.) \dot{r}_{11} & w_{2}(.) \dot{r}_{12} & \ldots & w_{n}(.) \dot{r}_{1 n} \\
w_{1}(.) \dot{r}_{21} & w_{2}(.) \dot{r}_{22} & \ldots & w_{n}(.) \dot{r}_{2 n} \\
\vdots & \ldots & \ldots & \ldots \\
\dot{w}_{1}(.) \dot{r}_{m 1} & \dot{w}_{2}(.) \dot{r}_{m 2} & \ldots & \dot{w}_{n}(.) \dot{r}_{m n}
\end{array}\right]}
\end{gathered}
$$

Step 7: Determination of FPIS and FNIS:

Equations are used to find the FPIS and the FNIS are given below.

$$
\begin{gathered}
A^{*}=\left(\grave{v}_{1}^{*}, \grave{v}_{2}^{*}, \ldots \ldots, \grave{v}_{n}^{*}\right) \text { where } \grave{v}_{j}^{*}=\left(c_{j}^{*}, c_{j}^{*}, c_{j}^{*}\right) \text { and } c_{j}^{*}= \\
\max _{i}\left\{\grave{v}_{i j}^{(3 r d \text { component })}\right. \\
A^{-}=\left(\grave{v}_{1}^{-}, \grave{v}_{2}^{-}, \ldots \ldots, \grave{v}_{n}^{-}\right) \text {where } \grave{v}_{j}^{-}=\left(c_{j}^{-}, c_{j}^{-}, c_{j}^{-}\right) \text {and } c_{j}^{-}=
\end{gathered}
$$

$$
\begin{gathered}
\min _{i}\left\{\grave{v}_{i j}^{(1 \text { st component })}\right. \\
\mathrm{i}=1,2,3, \ldots \ldots \ldots, \mathrm{m}, \mathrm{j}=1,2,3, \ldots \ldots \ldots \ldots, \mathrm{n}
\end{gathered}
$$

Step 8: Calculation of distances $d_{i}^{*}$ and $d_{i}^{-}$:

The distances $d_{i}^{*}$ and $d_{i}^{-}$of each weighted alternative $v_{i j}$; $1,2, \ldots, \mathrm{m}$ from FPIS and FNIS are calculated by using the distance formulas.

$$
\begin{aligned}
& d_{i}^{*}=\sum_{j=1}^{n} d\left(\dot{v}_{i j}, \grave{v}_{i}^{*}\right) \text { where } \mathrm{i}=1,2,3, \ldots \ldots \ldots, \mathrm{m} \\
& d_{i}^{-}=\sum_{j=1}^{n} d\left(\dot{v}_{i j}, \grave{v}_{i}^{-}\right) \text {where } \mathrm{i}=1,2,3, \ldots \ldots \ldots, \mathrm{m}
\end{aligned}
$$

where $d_{i}$ is the distance between two fuzzy numbers.

Step 9: Determination of Closeness Coefficient $C C_{i}$ :

In order to rank the alternatives closeness coefficient is computed by the following equation.

$$
\mathrm{CC}_{\mathrm{i}}=\frac{d_{i}^{-}}{d_{i}^{*}+d_{i}^{-}}, \text {for all } \mathrm{i}=1,2,3, \ldots \ldots \ldots ., \mathrm{m}
$$

Step 10: Ranking the alternatives:

The alternatives are ranked according to the fact that "an alternative with $\mathrm{CCi}$ closer to 1 indicates that the alternative is close to the FPIS and distant form FNIS. A large value of closeness index indicates a good performance of the alternative [24].

\section{Application of Fuzzy Topsis}

\subsection{Problem Scenario}

A company Alpha has to choose a best one from two alternatives $A_{1}$ and $A_{2}$. For the selection of best alternative, the company hire a team of decision makers which consist on three members, $\mathrm{D}=\left\{\mathrm{DM}_{1}, \mathrm{DM}_{2}, \mathrm{DM}_{3}\right\}$. Four evaluation criteria's are mentioned for the selection of alternative which are given below.

Four Evaluation Criteria $(n=4)$ represented by

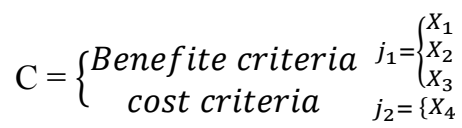

\subsection{Solution by Fuzzy Topsis}

Step 1: Selection of a Fuzzy Ratings Scale for Linguistic Variables

Rating scale for linguistic variables is given below in table 1 .

Table 1. Fuzzy Ratings for Linguistic Variables.

\begin{tabular}{lll}
\hline Criteria Weights & Alternatives & TFN \\
\hline Very Low (VL) & Very Poor (VP) & $(1,1,3)$ \\
Low (L) & Poor (P) & $(1,3,5)$ \\
Medium (M) & Fair (F) & $(3,5,7)$ \\
High (H) & Good (G) & $(5,7,9)$ \\
Very High (VH) & Very Good (VG) & $(7,9,9)$ \\
\hline
\end{tabular}

Step 2: Criteria weightage and fuzzy linguistic ratings for the alternatives given by DM's

In order to integrate the opinions of all the DMs, each DM allocates some weights to the criteria, given in the table 2. 
Table 2. Criteria Weightage by the DMs $\grave{w}_{j}^{k}=\left\{w_{j 1}^{k}, w_{j 2}^{k}, w_{j 3}^{k}\right\}$.

\begin{tabular}{llll}
\hline & $\mathbf{D M}_{1}$ & $\mathbf{D M}_{2}$ & $\mathbf{D M}_{3}$ \\
\hline $\mathrm{C}_{1}$ & $\mathrm{H}(5,7,9)$ & $\mathrm{M}(3,5,7)$ & $\mathrm{M}(3,5,7)$ \\
& $\grave{\mathrm{w}}_{1}^{1}=\left(\mathrm{w}_{11}^{1}, \mathrm{w}_{12}^{1}, \mathrm{w}_{13}^{1}\right)$ & $\grave{\mathrm{w}}_{1}^{2}=\left(\mathrm{w}_{11}^{2}, \mathrm{w}_{12}^{2}, \mathrm{w}_{13}^{2}\right)$ & $\grave{\mathrm{w}}_{1}^{3}=\left(\mathrm{w}_{11}^{3}, \mathrm{w}_{12}^{3}, \mathrm{w}_{13}^{3}\right)$ \\
$\mathrm{C}_{2}$ & $\mathrm{VH}(7,9,9)$ & $\mathrm{H}(5,7,9)$ & $\mathrm{H}(5,7,9)$ \\
& $\grave{\mathrm{w}}_{2}^{1}=\left(\mathrm{w}_{21}^{1}, \mathrm{w}_{22}^{1}, \mathrm{w}_{23}^{1}\right)$ & $\grave{\mathrm{w}}_{2}^{2}=\left(\mathrm{w}_{21}^{2}, \mathrm{w}_{22}^{2}, \mathrm{w}_{23}^{2}\right)$ & $\grave{\mathrm{w}}_{2}^{3}=\left(\mathrm{w}_{21}^{3}, \mathrm{w}_{22}^{3}, \mathrm{w}_{23}^{3}\right)$ \\
$\mathrm{C}_{3}$ & $\mathrm{VH}(7,9,9)$ & $\mathrm{H}(5,7,9)$ & $\mathrm{H}(5,7,9)$ \\
& $\grave{\mathrm{w}}_{3}^{1}=\left(\mathrm{w}_{31}^{1}, \mathrm{w}_{32}^{1}, \mathrm{w}_{33}^{1}\right)$ & $\grave{\mathrm{w}}_{3}^{2}=\left(\mathrm{w}_{31}^{2}, \mathrm{w}_{32}^{2}, \mathrm{w}_{33}^{2}\right)$ & $\grave{\mathrm{w}}_{3}^{3}=\left(\mathrm{w}_{31}^{3}, \mathrm{w}_{32}^{3}, \mathrm{w}_{33}^{3}\right)$ \\
$\mathrm{C}_{4}$ & $\mathrm{M}(3,5,7)$ & $\mathrm{L}(1,3,5)$ & $\mathrm{L}(1,3,5)$ \\
& $\grave{\mathrm{w}}_{4}^{1}=\left(\mathrm{w}_{41}^{1}, \mathrm{w}_{42}^{1}, \mathrm{w}_{43}^{1}\right)$ & $\grave{\mathrm{w}}_{4}^{2}=\left(\mathrm{w}_{41}^{2}, \mathrm{w}_{42}^{2}, \mathrm{w}_{43}^{2}\right)$ & $\grave{\mathrm{w}}_{4}^{3}=\left(\mathrm{w}_{41}^{3}, \mathrm{w}_{42}^{3}, \mathrm{w}_{43}^{3}\right)$ \\
\hline
\end{tabular}

Table 3 shows the aggregated fuzzy weights $w_{j}=\left(\dot{w}_{j 1}, \dot{w}_{j 2}, \dot{w}_{j 3}\right)$ for each criteria $(j=1,2,3,4)$.

Table 3. The aggregated fuzzy weights $\dot{w}_{j}=\left(\dot{w}_{j 1}, \dot{w}_{j 2}, \dot{w}_{j 3}\right)$ for each criteria $(j=1,2,3,4)$ are calculated.

\begin{tabular}{ll}
\hline$\dot{w}_{1}=\left(\dot{w}_{11}, \dot{w}_{12}, \dot{w}_{13}\right)=(3,5.667,9)$ & Where $w_{11}=\min _{k}\left\{w_{11}^{k}\right\}=\min \{5,3,3\}=3$ \\
$\dot{w}_{2}=\left(\dot{w}_{21}, \dot{w}_{22}, \dot{w}_{23}\right)=(5,7.667,9)$ & $w_{12}=\frac{1}{3} \sum_{k=1}^{3} w_{12}^{k}=\frac{1}{3}[1,7+5+5]=5.667$ \\
$\dot{w}_{3}=\left(\dot{w}_{31}, \dot{w}_{32}, \dot{w}_{33}\right)=(5,7.667,9)$ & $w_{13}=k \max \left\{w_{13}^{k}\right\}=\max \{9,7,7\}=9$ \\
$\dot{w}_{4}=\left(\dot{w}_{41}, \dot{w}_{42}, \dot{w}_{43}\right)=(1,3.667,7)$ & Similarly $\dot{w}_{2}, \dot{w}_{3}$ and $\dot{w}_{4}$ are calculated \\
\hline
\end{tabular}

$$
\begin{aligned}
\grave{W} & =\left[\dot{w}_{1}, \dot{w}_{2}, \dot{w}_{3}, \dot{w}_{4}\right]^{\text {Transpose }} \\
& =[(3,5.667,9),(5,7.667,9),(5,7.667,9),(1,3.667,7)]^{\text {Transpose }}
\end{aligned}
$$

The alternative ratings, according to the DM's are listed in the below table 4 .

\begin{tabular}{|c|c|c|c|c|}
\hline \multirow{2}{*}{ Cri. } & \multirow{2}{*}{ Alts. } & \multicolumn{3}{|l|}{ Decision Makers } \\
\hline & & $\mathrm{DM}_{1}$ & $\mathbf{D M}_{2}$ & $\mathbf{D M}_{33}$ \\
\hline \multirow{4}{*}{$\mathrm{C}_{1}$} & $A_{1}$ & $\mathrm{~F}$ & $\mathrm{~F}$ & $\mathrm{~F}$ \\
\hline & & $\breve{\mathrm{x}}_{11}^{1}=\left(\mathrm{a}_{11}^{1}, \mathrm{~b}_{11}^{1}, \mathrm{c}_{11}^{1}\right)=(3,5,7)$ & $\breve{x}_{11}^{2}=\left(a_{11}^{2}, b_{11}^{2}, c_{11}^{2}\right)=(3,5,7)$ & $\breve{x}_{11}^{3}=\left(a_{11}^{3}, b_{11}^{3}, c_{11}^{3}\right)=(3,5,7)$ \\
\hline & $A_{1}$ & $\mathrm{G}$ & & \\
\hline & $A_{2}$ & $\check{\mathrm{x}}_{21}^{1}=\left(\mathrm{a}_{21}^{1}, \mathrm{~b}_{21}^{1}, \mathrm{c}_{21}^{1}\right)=(5,7,9)$ & $\breve{\mathrm{x}}_{21}^{2}=\left(\mathrm{a}_{21}^{2}, \mathrm{~b}_{21}^{2}, \mathrm{c}_{21}^{2}\right)=(5,7,9)$ & $\breve{\mathrm{x}}_{21}^{3}=\left(\mathrm{a}_{21}^{3}, \mathrm{~b}_{21}^{3}, \mathrm{c}_{21}^{3}\right)=(3,5,7)$ \\
\hline \multirow{3}{*}{$\mathrm{C}_{2}$} & $\mathrm{~A}_{1}$ & $\begin{array}{l}\text { VG } \\
\breve{x}_{12}^{1}=\left(a_{12}^{1}, b_{12}^{1}, c_{12}^{1}\right)=(7,9,9)\end{array}$ & $\begin{array}{l}\text { VG } \\
\breve{x}_{12}^{2}=\left(a_{12}^{2}, b_{12}^{2}, c_{12}^{2}\right)=(7,9,9)\end{array}$ & $\begin{array}{l}\mathrm{VG} \\
\breve{\mathrm{x}}_{12}^{1}=\left(\mathrm{a}_{12}^{3}, \mathrm{~b}_{12}^{3}, \mathrm{c}_{12}^{3}\right)=(7,9,9)\end{array}$ \\
\hline & & $\begin{array}{l}12 \\
G\end{array}$ & $\begin{array}{l}\mathrm{A}_{12} \\
\mathrm{VG}\end{array}$ & \\
\hline & $\mathrm{A}_{2}$ & $\breve{x}_{22}^{1}=\left(a_{22}^{1}, b_{22}^{1}, c_{22}^{1}\right)=(5,7,9)$ & $\breve{\mathrm{x}}_{22}^{2}=\left(\mathrm{a}_{22}^{2}, \mathrm{~b}_{22}^{2}, \mathrm{c}_{22}^{2}\right)=(3,5,7)$ & $\breve{\mathrm{x}}_{22}^{3}=\left(\mathrm{a}_{22}^{3}, \mathrm{~b}_{22}^{3}, \mathrm{c}_{22}^{3}\right)=(5,7,9)$ \\
\hline \multirow{3}{*}{$\mathrm{C}_{3}$} & $\mathrm{~A}_{1}$ & $\begin{array}{l}\mathrm{P} \\
\breve{\mathrm{x}}_{13}^{1}=\left(\mathrm{a}_{13}^{1}, \mathrm{~b}_{13}^{1}, \mathrm{c}_{13}^{1}\right)=(1,3,5)\end{array}$ & $\begin{array}{l}\mathrm{F} \\
\breve{\mathrm{x}}_{13}^{2}=\left(\mathrm{a}_{13}^{2}, \mathrm{~b}_{13}^{2}, \mathrm{c}_{13}^{2}\right)=(3,5,7)\end{array}$ & $\begin{array}{l}\mathrm{P} \\
\breve{\mathrm{x}}_{13}^{3}=\left(\mathrm{a}_{13}^{3}, \mathrm{~b}_{13}^{3}, \mathrm{c}_{13}^{3}\right)=(1,3,5)\end{array}$ \\
\hline & $A_{2}$ & P & & \\
\hline & $\mathrm{A}_{2}$ & $\check{x}_{23}^{1}=\left(a_{23}^{1}, b_{23}^{1}, c_{23}^{1}\right)=(1,3,5)$ & $\breve{x}_{23}^{2}=\left(a_{23}^{2}, b_{23}^{2}, c_{23}^{2}\right)=(1,3,5)$ & $\check{\mathrm{x}}_{23}^{3}=\left(\mathrm{a}_{23}^{3}, \mathrm{~b}_{23}^{3}, \mathrm{c}_{23}^{3}\right)=(1,3,5)$ \\
\hline \multirow{3}{*}{$\mathrm{C}_{4}$} & $\mathrm{~A}_{1}$ & $\begin{array}{l}\mathrm{F} \\
\breve{\mathrm{x}}_{14}^{1}=\left(\mathrm{a}_{14}^{1}, \mathrm{~b}_{14}^{1}, \mathrm{c}_{14}^{1}\right)=(3,5,7)\end{array}$ & $\begin{array}{l}\mathrm{F} \\
\breve{\mathrm{X}}_{14}^{2}=\left(\mathrm{a}_{1}^{2}, \mathrm{~b}_{14}^{2}, \mathrm{c}_{1+1}^{2}\right)=(3,5,7)\end{array}$ & $\begin{array}{l}\mathrm{P} \\
\breve{\mathrm{x}}_{14}^{3}=\left(\mathrm{a}^{3}, \mathrm{~b}_{1,}^{3}, \mathrm{c}_{14}^{3}\right)=(1,3,5)\end{array}$ \\
\hline & & & & \\
\hline & $\mathrm{A}_{2}$ & $\check{\mathrm{X}}_{24}^{1}=\left(\mathrm{a}_{24}^{1}, \mathrm{~b}_{24}^{1}, \mathrm{c}_{24}^{1}\right)=(1,3,5)$ & $\breve{x}_{24}^{2}=\left(a_{24}^{2}, b_{24}^{2}, c_{24}^{2}\right)=(1,3,5)$ & $\check{\mathrm{x}}_{24}^{3}=\left(\mathrm{a}_{24}^{3}, \mathrm{~b}_{24}^{3}, \mathrm{c}_{24}^{3}\right)=(3,5,7)$ \\
\hline
\end{tabular}

Table 4. Alternative Ratings $\breve{x}_{i j}^{k}=\left(a_{i j}^{k}, b_{i j}^{k}, c_{i j}^{k}\right)$.

Step 3: Compute the aggregated fuzzy ratings for the alternatives

Aggregated Fuzzy Ratings $\dot{x}_{i j}$ for alternatives are calculated by using given equations

$$
\begin{aligned}
& \dot{x}_{11}=\left(\mathrm{a}_{11}, \mathrm{~b}_{11}, \mathrm{c}_{11}\right) \\
& \mathrm{a}_{11}=\min _{k}\left\{a_{11}^{k}\right\}=\min \{3,3,3\}=3 \\
& b_{11}=\frac{1}{3} \sum_{k=1}^{3} b_{11}^{k}=\frac{1}{3}[5+5+5]=5
\end{aligned}
$$

$$
\mathrm{c}_{11}=\max _{k}\left\{c_{11}^{k}\right\}=\max \{7,7,7\}=7
$$

Therefore,

$$
\dot{x}_{11}=(3.000,5.000,7.000)
$$

Other values are calculated in a similar manner and are given in the below table 5.

Step-4: Construction of AFDM D̀ $=\left[\dot{x}_{i j}\right]$

Table 5. Construction of AFDM.

\begin{tabular}{lllll}
\hline & $\mathbf{C}_{1}$ & $\mathbf{C}_{2}$ & $\mathbf{C}_{3}$ & $\mathbf{C}_{4}$ \\
\hline $\mathrm{A}_{1}$ & $\dot{x}_{11}=(3.000,5.000,7.000)$ & $\dot{x}_{12}=(7.000,9.000,9.000)$ & $\dot{x}_{13}=(1.000,3.667,7.000)$ & $\dot{x}_{12}=(1.000,4.333,7.000)$ \\
$\mathrm{A}_{2}$ & $\dot{x}_{21}=(3.000,6.333,9.000)$ & $\dot{x}_{22}=(5.000,7.667,9.000)$ & $\dot{x}_{23}=(1.000,3.000,5.000)$ & $\dot{x}_{24}=(1.000,3.667,7.000)$ \\
\hline
\end{tabular}

Step 5: Normalization of the AFDM

The values for the NAFDM are calculated by using the equations which are given for benefit criteria and cost criteria. 
$\dot{r}_{11}=\left(\frac{a_{1}^{-}}{c_{11}}, \frac{a_{1}^{-}}{b_{11}}, \frac{a_{1}^{-}}{a_{11}}\right)$ where $\mathrm{C}_{1}$ is the cost criteria $=\left(\frac{3}{7}, \frac{3}{5}, \frac{3}{3}\right)$ where $a_{1}^{-}=\min _{i} a_{i 1}=\min \{3.000,3.000\}=3$ $\dot{r}_{21}=\left(\frac{a_{1}^{-}}{c_{21}}, \frac{a_{1}^{-}}{b_{21}}, \frac{a_{1}^{-}}{a_{21}}\right)$ where $C_{1}$ is the cost criteria $=\left(\frac{3}{9}, \frac{3}{6.33}, \frac{3}{3}\right)$ where $a_{1}^{-}=\min _{i} a_{i 1}=\min \{3.000$, $3.000\}=3$

$\dot{r}_{12}=\left(\begin{array}{lll}\frac{a_{12}}{c_{2}^{*}} & \frac{b_{12}}{c_{2}^{*}} & \frac{c_{12}}{c_{2}^{*}}\end{array}\right)$ where $\mathrm{C}_{2}$ is the benefit criteria $=\left(\frac{7}{9}, \frac{9}{9}, \frac{9}{9}\right)$ where $c_{2}^{*}=\max _{i} c_{i 2}=\max \{9.000,9.000\}$ $=9.000$

$\dot{r}_{22}=\left(\begin{array}{lll}\frac{a_{22}}{c_{2}^{*}} & \frac{b_{22}}{c_{2}^{*}} & \frac{c_{22}}{c_{2}^{*}}\end{array}\right)$ where $\mathrm{C}_{2}$ is the benefit criteria $=\left(\frac{5}{9}, \frac{7.667}{9}, \frac{9}{9}\right)$ where $c_{2}^{*}=\max _{i} c_{i 2}=\max \{9.000$, $9.000\}=9.000$ $\dot{r}_{13}=\left(\begin{array}{lll}\frac{a_{13}}{c_{3}^{*}} & \frac{b_{13}}{c_{3}^{*}} & \frac{c_{13}}{c_{3}^{*}}\end{array}\right)$ where $\mathrm{C}_{3}$ is the benefit criteria $=\left(\frac{1}{7}, \frac{3.667}{7}, \frac{7}{7}\right)$ where $c_{3}^{*}=\max _{i} c_{i 3}=\max \{7.000$, $5.000\}=7.000$

$\dot{r}_{23}=\left(\begin{array}{lll}\frac{a_{23}}{c_{3}^{*}} & \frac{b_{23}}{c_{3}^{*}} & \frac{c_{23}}{c_{3}^{*}}\end{array}\right)$ where $\mathrm{C}_{3}$ is the benefit criteria

$=\left(\frac{1}{7}, \frac{3}{7}, \frac{5}{7}\right)$ where $c_{3}^{*}=\max _{i} c_{i 3}=\max \{7.000,5.000\}$

$=7.000$

$\dot{r}_{14}=\left(\begin{array}{lll}\frac{a_{14}}{c_{4}^{*}} & \frac{b_{14}}{c_{4}^{*}} & \frac{c_{14}}{c_{4}^{*}}\end{array}\right)$ where $\mathrm{C}_{4}$ is the benefit criteria $=\left(\frac{1}{7}, \frac{3}{7}, \frac{5}{7}\right)$ where $c_{4}^{*}=\max _{i} c_{i 4}=\max \{7.000,7.000\}$ $=7.000$

$\dot{r}_{24}=\left(\begin{array}{lll}\frac{a_{24}}{c_{4}^{*}} & \frac{b_{24}}{c_{4}^{*}} & \frac{c_{24}}{c_{4}^{*}}\end{array}\right)$ where $\mathrm{C}_{4}$ is the benefit criteria $=\left(\frac{1}{7}, \frac{3.667}{7}, \frac{7}{7}\right)$ where $c_{4}^{*}={ }_{i}^{\max } c_{i 4}=\max \{7.000$, $7.000\}=7.000$

Therefore, Normalized Aggregated Fuzzy Decision Matrix (NAFDM) is tabulated in the given table 6.

Table 6. $N A F D M \grave{R}=\left[\dot{r}_{i j}\right]$.

\begin{tabular}{lllll}
\hline & $\boldsymbol{C}_{\boldsymbol{I}}$ & $\boldsymbol{C}_{2}$ & $\boldsymbol{C}_{3}$ & $\boldsymbol{C}_{4}$ \\
\hline $\mathrm{A}_{1}$ & $\dot{r}_{11}=(0.429,0.600,1.000)$ & $\dot{r}_{12}=(0.778,1.000,1.000)$ & $\dot{r}_{13}=(0.143,0.524,1.000)$ & $\dot{r}_{14}=(0.143,0.619,1.000)$ \\
$\mathrm{A}_{2}$ & $\dot{r}_{21}=(0.333,0.474,1.000)$ & $\dot{r}_{22}=(0.556,0.852,1.000)$ & $\dot{r}_{23}=(0.143,0.429,0.714)$ & $\dot{r}_{24}=(0.143,0.524,1.000)$ \\
\hline
\end{tabular}

Step 6: WNFDM

To get WNFDM, the values from above table are multiplied with AWM which are calculated in table 7.

Table 7. $W N F D M \grave{V}=\left[\hat{v}_{i j}\right]$.

\begin{tabular}{lllll}
\hline & $\mathbf{C}_{1}$ & $\mathbf{C}_{2}$ & $\mathbf{C}_{3}$ & $\mathbf{C}_{4}$ \\
\hline $\mathrm{A}_{1}$ & $\dot{v}_{11}=(1.286,3.400,9.000)$ & $\dot{v}_{12}=(3.889,7.667,9.000)$ & $\dot{v}_{13}=(0.714,4.016,9.000)$ & $\dot{v}_{14}=(0.143,2.270,7.000)$ \\
$\mathrm{A}_{2}$ & $\dot{v}_{21}=(1.000,2.684,9.000)$ & $\dot{v}_{22}=(2.778,6.531,9.000)$ & $\dot{v}_{23}=(0.714,3.286,6.429)$ & $\dot{v}_{24}=(0.143,1.921,7.000)$ \\
\hline
\end{tabular}

Step 7: Determination of FPIS and FNIS

Table 8 gives the calculated values of FPIS and FNIS respectively.

Table 8. Calculated values of FPIS and FNIS.

\begin{tabular}{lllll}
\hline $\mathrm{A}^{*}$ & $v_{1}^{*}=(9,9,9)$ & $v_{2}^{*}=(9,9,9)$ & $v_{3}^{*}=(9,9,9)$ & $v_{4}^{*}=(7,7,7)$ \\
$A^{-}$ & $v_{1}^{-}=(1,1,1)$ & $v_{2}^{-}=(2.778,2.778,2.778)$ & $v_{3}^{-}=(0.714,0.714,0.714)$ & $v_{4}^{-}=(0.143,0.143,0.143)$ \\
\hline
\end{tabular}

Step 8: Calculation of distances $d_{i}^{*}$ and $d_{i}^{-}$

To find the distances $d_{i}^{*}$ and $d_{i}^{-}$, it is necessary to find out the distance of each weighted normalized alternative from the FPIS and FNIS respectively.

$$
\begin{aligned}
& \mathrm{d}\left(v_{1 j}, v_{j}^{*}\right) \mathrm{j}=1,2,3,4 \\
& \mathrm{~d}\left(v_{11}, v_{1}^{*}\right)=\mathrm{d}((1.286,3.400,9.000),(9,9,9)) \text { for } \mathrm{j}=1 \\
& =\sqrt{\frac{1}{3} \quad\left((1.287-9)^{2}+(3.400-9)^{2}+(9.000-9)^{2}\right)} \\
& =5.503 \\
& \mathrm{~d}\left(v_{12}, v_{2}^{*}\right)=\mathrm{d}((3.889,7.667,9.000),(9,9,9)) \text { for } \mathrm{j}=2 \\
& =\sqrt{\frac{1}{3} \quad\left((3.889-9)^{2}+(7.667-9)^{2}+(9.000-9)^{2}\right)} \\
& =3.049
\end{aligned}
$$

$$
\begin{aligned}
& \mathrm{d}\left(v_{13}, v_{3}^{*}\right)=\mathrm{d}((0.714,4.016,9.000),(9,9,9)) \text { for } \mathrm{j}=3 \\
& =\sqrt{\frac{1}{3}\left((0.714-9)^{2}+(4.016-9)^{2}+(9.000-9)^{2}\right)} \\
& =5.582 \\
& \mathrm{~d}\left(v_{14}, v_{4}^{*}\right)=\mathrm{d}((0.143,2.270,7.000),(7,7,7)) \text { for } \mathrm{j}=4 \\
& =\sqrt{\frac{1}{3}\left((0.143-7)^{2}+(2.270-7)^{2}+(7.000-7)^{2}\right)} \\
& =4.809
\end{aligned}
$$

The remaining values $d\left(v_{2 j}, v_{j}^{*}\right), d\left(v_{1 j}, v_{j}^{-}\right), d\left(v_{2 j}, v_{j}^{-}\right)$ for $j=1,2,3,4$, are left for the sake of brevity.

In table 9 the distances $d\left(A_{i}, A^{*}\right)$ and $d\left(A_{i}, A^{-}\right)$from FPIS and FNIS for the Alternatives $A_{i}$ are calculated. 
Table 9. Distances $d\left(A_{i}, A^{*}\right)$ and $d\left(A_{i}, A^{-}\right)$from FPIS and FNIS for the Alternatives $A_{i}$.

\begin{tabular}{lllll}
\hline & $\mathbf{C}_{1}$ & $\mathbf{C}_{2}$ & $\mathbf{C}_{3}$ & $\mathbf{C}_{4}$ \\
\hline FPIS A $_{1}$ & $\mathrm{~d}\left(v_{11}, v_{1}^{*}\right)=5.503$ & $\mathrm{~d}\left(v_{12}, v_{2}^{*}\right)=3.049$ & $\mathrm{~d}\left(v_{13}, v_{3}^{*}\right)=5.582$ & $\mathrm{~d}\left(v_{14}, v_{4}^{*}\right)=4.809$ \\
FPIS A $_{2}$ & $\mathrm{~d}\left(v_{21}, v_{1}^{*}\right)=5.884$ & $\mathrm{~d}\left(v_{22}, v_{2}^{*}\right)=3.864$ & $\mathrm{~d}\left(v_{23}, v_{3}^{*}\right)=5.997$ & $\mathrm{~d}\left(v_{24}, v_{4}^{*}\right)=4.926$ \\
FNIS A $_{1}$ & $\mathrm{~d}\left(v_{11}, v_{1}^{-}\right)=4.824$ & $\mathrm{~d}\left(v_{12}, v_{2}^{-}\right)=4.613$ & $\mathrm{~d}\left(v_{13}, v_{3}^{-}\right)=5.149$ & $\mathrm{~d}\left(v_{14}, v_{4}^{-}\right)=4.145$ \\
FNIS A $_{2}$ & $\mathrm{~d}\left(v_{21}, v_{1}^{-}\right)=4.72$ & $\mathrm{~d}\left(v_{22}, v_{2}^{-}\right)=4.195$ & $\mathrm{~d}\left(v_{23}, v_{3}^{-}\right)=3.617$ & $\mathrm{~d}\left(v_{24}, v_{4}^{-}\right)=4.089$ \\
\hline
\end{tabular}

The distance $d_{i}^{*}$ of each weighted alternative from FPIS is computed using

$$
d_{i}^{*}=\sum_{j=1}^{n} d\left(v_{i j}, v_{j}^{*}\right): \mathrm{i}=1,2
$$

Now $d_{i}^{*}$ for the alternative $\mathrm{A}_{1}$ form FPIS $\mathrm{A}^{*}$ is calculated by using this equation

$$
\begin{gathered}
d_{1}^{*}=\sum_{j=1}^{n} d\left(v_{1 j}, v_{j}^{*}\right) \\
=d\left(v_{11}, v_{1}^{*}\right)+d\left(v_{12}, v_{2}^{*}\right)+d\left(v_{13}, v_{3}^{*}\right)+d\left(v_{14}, v_{4}^{*}\right)
\end{gathered}
$$

Putting the values in above equation from above table.

$$
\begin{gathered}
d_{1}^{*}=d\left(v_{11}, v_{1}^{*}\right)+d\left(v_{12}, v_{2}^{*}\right)+d\left(v_{13}, v_{3}^{*}\right)+d\left(v_{14}, v_{4}^{*}\right) \\
=5.503+3.049+5.582+4.809 \\
=18.943
\end{gathered}
$$

Similarly $d_{2}^{*}, d_{1}^{-}$and $d_{2}^{-}$are calculated but for the sake of brevity only their respective values are given in the table 10 .

Table 10. The distance of each weighted alternative.

\begin{tabular}{llll}
\hline $\boldsymbol{d}_{\mathbf{1}}^{*}$ & $\boldsymbol{d}_{\mathbf{2}}^{*}$ & $\boldsymbol{d}_{\mathbf{1}}^{-}$ & $\boldsymbol{d}_{\mathbf{2}}^{-}$ \\
\hline 18.943 & 20.671 & 18.731 & 16.621 \\
\hline
\end{tabular}

Step 9: Determination of Closeness Coefficient $C_{i}$

Finally the closeness coefficient $\mathrm{CCi}$ of each alternative (i $=1,2$ ) is calculated by using the formula

$$
\begin{aligned}
& \mathrm{CCi}=\frac{d_{i}^{-}}{d_{i}^{-}+d_{i}^{*}} \text { as follows } \\
& \mathrm{CC}_{1}=\frac{18.731}{18.731+18.943}=0.497 \\
& \mathrm{CC}_{2}=\frac{16.621}{16.621+20.671}=0.445
\end{aligned}
$$

Step 10: Ranking the alternatives

Hence the ranking order for the alternatives is $A_{1}>A_{2}$, i.e., the best choice considering the given criteria.

\section{Conclusion}

Classical TOPSIS uses crisp techniques for the linguistic assessments, but due to imprecise and fuzziness nature of the linguistic assessments, we faced some problems to find out the solution of these problems we proposed the Fuzzy TOPSIS with its example. It is evident that fuzzy TOPSIS has the ability to deal situations where ambiguity occurs due to the presence of linguistic variables, whereas Classical TOPSIS is lacking this property.

\section{Acknowledgements}

The authors are very grateful to the editor and reviewers for their comments and suggestions, which are helpful in improving the paper.

\section{References}

[1] Hwang C, Yoon K, "Multiple Attribute Decision Making: Methods and Applications", A State of the Art Survey, 1 (1981).

[2] Chen S. J. J, Hwang C. L, "Fuzzy Multiple Attribute Decision Making Methods", 375 (1992).

[3] Chen, C. T. "Extensions of the TOPSIS for group decisionmaking under fuzzy environment", Fuzzy Sets Syst, 114 (1): $1-9(2000)$.

[4] Awasthi A, Chauhan S. S, Goyal S. K, "A multi-criteria decision making approach for location planning for urban distribution centers under uncertainty", Math. Comput. Model, 53 (1-2): 98-109 (2011).

[5] Chu, T. C. "Selecting plant location via a fuzzy TOPSIS approach", Int. J. Adv. Manuf. Techno, 20 (11): 859-864 (2002).

[6] Yong, D. "Plant location selection based on fuzzy TOPSIS", Int. J. Adv. Manuf. Technol, 28 (7-8): 839-844 (2006).

[7] Wang Y. M, Elhag T. M. S, "Fuzzy TOPSIS method based on alpha level sets with an application to bridge risk assessment", Expert Syst. Appl., 31 (2): 309-319 (2006).

[8] Dong W. M, Wong F. S, "Fuzzy Weighted Averages and Implementation of the Extension Principle", 21: 183-199 (1987).

[9] Mahmoodzadeh S., Shahrabi J., Pariazar M., and Zaeri M. S., "Project selection by using fuzzy AHP and TOPSIS technique", Int. J. Soc. Manag. Econ. Bus. Eng, 1 (3): 324 329 (2007).

[10] Yong - tao T., Shen L., Langston C. and Liu Li., "Construction project selection using fuzzy TOPSIS approach", J. Model. Manag, 5 (3): 302-315 (2010).

[11] Zadeh L. A. "The concept of a linguistic variable and its application to approximate reasoning-I", Inf. Sci. (Ny), 8 (3): 199-249 (1975).

[12] Saremi H. Q and Montazer G. A, "An application of type-2 fuzzy notions in website structures selection Utilizing extended TOPSIS method", WSEAS Trans. Comput, 7 (1): 8$15(2008)$ 
[13] Chen T. Y., Tsao C. Y, "The interval-valued fuzzy TOPSIS method and experimental analysis", Fuzzy Sets Syst, 159 (11): $1410-1428$ (2008).

[14] Zulqarnain M. and Saeed M., "An application of Interval valued fuzzy soft matrix (IVFSM) in decision making", Sci. Int. (Lahore), 28(3): 2261-2264 (2016).

[15] Zulqarnain M. and Saeed M., "Comparison between fuzzy soft matrix (FSM) and interval valued fuzzy soft matrix (IVFSM) in decision making", Sci. Int. (Lahore), 28(5): 42774283(2016).

[16] Zulqarnain M. and Saeed M., "A New Decision Making Method on Interval Valued Fuzzy Soft Matrix (IVFSM)", British Journal of Mathematics \& Computer Science, 20(5): 117(2017).

[17] Ashtiani B., Haghighirad F., Makui A. and Ali Montazer G., "Extension of fuzzy TOPSIS method based on interval-valued fuzzy sets", Appl. Soft Comput. J, 9 (2): 457-461 (2009).

[18] BOROVIČKA, A. "Fuzzy weights estimation method based on the linguistic expression of criteria relevance Fuzzy weights estimation method based on the linguistic expression of criteria relevance", (2014).
[19] Madi E. N., Garibaldi J. M. and Wagner C., "A comparison between two types of Fuzzy TOPSIS Method A comparison between two types of Fuzzy TOPSIS Method", Res. Gate, (2015).

[20] Ashrafzadeh M., Rafiei F. M., Isfahani N. M. Zare Z., "Application of fuzzy TOPSIS method for the selection of Warehouse Location", A Case Study," Interdiscip. J. Contemp. Res. Bus, 3: 655-671 (2012).

[21] Cheng D. Y., Chao K. M., Lo C. C. and Tsai C. F., "A user centric service-oriented modeling approach", World Wide Web. 14 (4): 431-459 (2011).

[22] Zadeh, L. A. "Fuzzy Sets", Journal of Information and Control, 8(3): 338-353 (1965).

[23] B. Sodhi and P. T., "A Simplified Description of Fuzzy TOPSIS” 6-8 (2012).

[24] R. Gupta, A. Sachdeva, and A. Bhardwaj, "Selection of 3pl service provider using integrated fuzzy delphi and fuzzy TOPSIS" Proc. World Congr. Eng. Comput. Sci, 2 20-22 (2010). 